\title{
MODELO CONCEITUAL PARA CRIAÇÃO, APLICAÇÃO E AVALIAÇÃO DE JOGOS EDUCATIVOS DIGITAIS
}

\author{
Rafael Feyh Jappur - Faculdade Senac Florianópolis - rjappur@ gmail.com \\ Fernando Jose Spanhol - UFSC - profspanhol@ gmail.com \\ Fernando Antonio Forcellini - UFSC - forcellini@deps.ufsc.br
}

\begin{abstract}
Resumo: Os jogos educativos digitais têm o potencial de agregar valor ao processo de ensino e aprendizagem de conteúdos em sala de aula. Todavia, os professores ou mediadores desse processo enfrentam dificuldades para a aplicação desses jogos no contexto da sala de aula. Nesse sentido, este trabalho buscou desenvolver um modelo conceitual para a criação, aplicação e avaliação de jogos educativos digitais para o contexto do processo de ensino e aprendizagem em sala de aula. Os procedimentos metodológicos utilizados foram preponderantemente a pesquisa bibliográfica e de campo, com a utilização do Design Science Research Methodology (DSRM) para o desenvolvimento do modelo conceitual. $O$ modelo conceitual foi aplicado com o uso do jogo educativo digital denominado de Simulador Ambiental. Constatou-se que houve melhora na percepção dos alunos quanto ao seu nível de aprendizado no conteúdo do jogo. Portanto, os resultados demonstraram positivamente a consistência do modelo conceitual, oferecendo às partes interessadas no processo de ensino e aprendizagem com o uso de jogos educativos digitais em sala de aula, um caminho pelo qual eles possam criar, aplicar e avaliar a qualidade dos jogos e a didática de aplicação destes.
\end{abstract}

Palavras-chave: jogos educativos digitais, processo de ensino e aprendizagem, educação, sustentabilidade.

\section{Conceptual Model for Creation, Implementation and Evaluation of digital educational games}

\begin{abstract}
Digital educational games have the potential to add value to the process of teaching and learning classroom content. However, teachers or mediators of this process facing difficulties in the application of these games in the context of the classroom. Thus, this study sought to develop a conceptual model for the creation, implementation and evaluation of digital educational games for the context of teaching and learning process in the classroom. The methodological procedures used were mainly bibliographical and field research, using the Design Science Research Methodology (DSRM) for the development of the conceptual model. The conceptual model was applied with the use of digital educational game called Environmental Simulator. It was found that there was an improvement in the perception of students about their learning level game content. Therefore, the results showed positive consistency of the conceptual model, offering stakeholders in the process of teaching and learning with the use of digital educational games in the classroom, a way by which they can create, implement and evaluate the quality of the games and the didactic application of these.
\end{abstract}

Keywords: digital educational games, teaching and learning process, education, sustainability. 


\section{Introdução}

$\mathrm{O}$ artigo aborda o contexto do uso de jogos educativos digitais no processo de ensino e aprendizagem em sala de aula. Os jogos educativos digitais podem ser um agregador para o ensino de conteúdos e para o processo de aprendizagem em sala de aula. Todavia, constata-se - conforme apresentam Kirriemuir e Mcfarlane (2004); Balasubramanian e Wilson, (2006); Baek, (2008), Echeverría et al. (2011), entre outros - que os professores ou mediadores possuem dificuldades para aplicar os jogos em sala de aula.

Estas dificuldades, conforme Balasubramanian e Wilson (2006), repousam na baixa utilização dos jogos educativos digitais nas escolas e, para muitos educadores, o desafio é encontrar e utilizar bons jogos como ferramenta de aprendizagem. Para os autores, os jogos digitais, no seu processo de criação têm sido usados de forma não articulada aos princípios e necessidades pedagógicas. Isto muitas vezes gera fragilidades ou dificuldades no que tange à seleção e aplicação pelos educadores, por não compreenderem o valor agregado à aprendizagem. Constata-se, ainda, que além da falta dos princípios pedagógicos na criação dos jogos, outras dificuldades são verificadas no uso do jogo em sala de aula, pela falta de mediação na aplicação, ou até mesmo da avaliação da eficiência e eficácia do uso dos jogos em sala de aula (KIRRIEMUIR, MCFARLANE, 2004; BALASUBRAMANIAN, WILSON, 2006; BAEK, 2008; ECHEVERRÍA, et al., 2011).

O problema de modelar jogos educativos digitais é salientado por alguns autores, tais como Hsiao (2007), Moreno-ger et al. (2008), Aleven et al. (2010), Echeverría et al. (2011), Villalta et al. (2011), Savi (2011), entre outros. Porém, verifica-se na literatura consultada que existem lacunas de como criar, aplicar e avaliar os jogos educativos digitais para o contexto do processo de ensino e aprendizagem em sala de aula. Também se verificou que os modelos pesquisados estão mais ligados à criação e recentemente com a avaliação dos jogos. Já no contexto da aplicação, a maioria dos modelos pesquisados focam na descrição dos processos de validação dos jogos, do que sobre a mediação da prática pedagógica em si (HSIAO, 2007; SAVI, 2011).

O presente artigo, portanto, pretende apresentar uma proposta de modelo conceitual que estruture de forma integrada os processos para a criação, aplicação e avaliação de jogos educativos digitais, no contexto da sala de aula, tendo como foco de análise a aplicação de um jogo educativo digital denominado de Simulador Ambiental (SA), nos programas Pronatec e Jovens Aprendizes da Faculdade Senac Florianópolis.

Na sequência, apresenta-se uma breve descrição da metodologia utilizada, a proposta de modelo conceitual e os resultados e discuções sobre a aplicação e avaliação do mesmo, terminando com as considerações finais.

\section{Metodologia}

A caracterização metodológica desta pesquisa, segundo Vergara (2009) e Gil (2010), possui mais similaridades com a pesquisa aplicada; quanto à forma de abordagem, representa uma pesquisa qualitativa; quanto aos objetivos, situa-se em três categorias: exploratória, descritiva e explicativa, os tipos de pesquisa não são mutuamente exclusivos, o que permite classificá-lo nestes três tipos. Em relação aos 
procedimentos adotados para a coleta de dados, esta pesquisa foi composta preponderantemente da pesquisa bibliográfica (pesquisas bibliométricas e exploratórias) e da pesquisa de campo, como parte prática para a coleta de dados (uso de protocolos, questionários e de fichas técnicas para anotações e registros de campo).

Ressalta-se, ainda, o uso do Design Science Research Methodology (DSRM) de Peffers et al. (2007), como procedimento metodológico da pesquisa para o desenvolvimento do modelo conceitual, que será descrito na próxima seção. O início desta pesquisa, conforme orienta o DSRM, foi o projeto e desenvolvimento do jogo educativo digital denominado de Simulador Ambiental (SA) (acesso www.meensina.org.br/site/simulador/2. A partir da experiência realizada com a criação do jogo que foi concebido o presente modelo conceitual.

\section{A PROPOSTA DE MODELO CONCEITUAL}

A proposta de modelo conceitual visa estabelecer uma estrutura de processos para a criação, aplicação e avaliação de jogos educativos digitais no processo de ensino e aprendizagem em sala de aula (Figura 1).

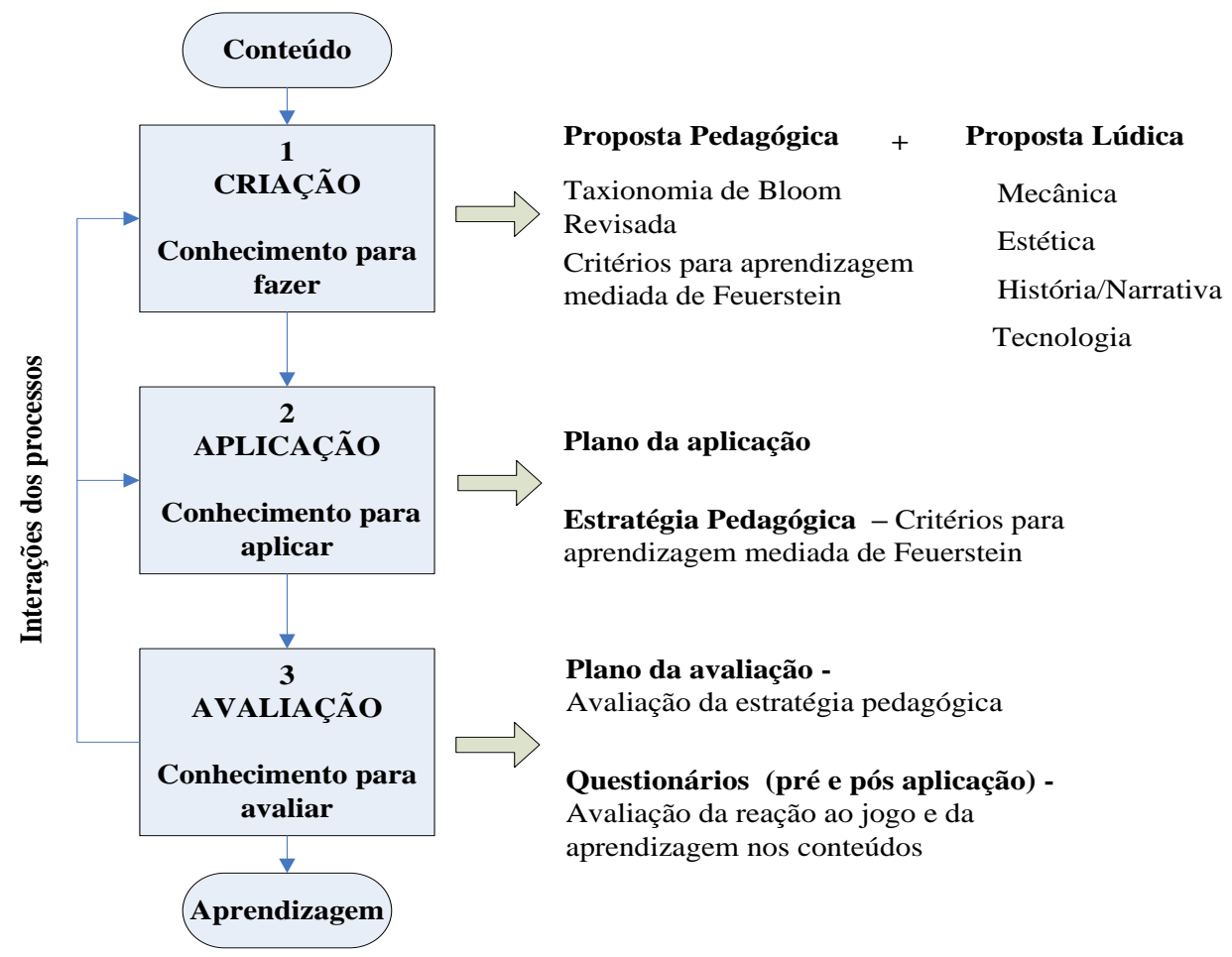

Figura 1. Modelo conceitual para jogos educativos digitais. Fonte: Autores.

O processo de criação envolve uma proposta pedagógica e lúdica para que os desenvolvedores criem bons jogos educativos digitais. A estrutura pedagógica propõe a utilização da taxionomia de Bloom (1977), porém na versão revisada conforme descrevem Anderson et al. (2001), Krathwohl (2002), Mayer (2002), Aleven et al. (2010), Villalta et al. (2011) e Echeverría et al. (2011) para a definição dos objetivos educacionais do jogo e de um guia de recomendações com os critérios de mediação de Feuerstein (1980), em conformidade com The International Center for the Enhancement 
of Learning Potential (ICELP) (2012) e Gomes (2001), adaptados para o contexto de criação dos jogos educativos digitais.

Já a proposta lúdica busca estabelecer um guia de recomendações com os elementos lúdicos a serem incorporados nos jogos. O guia lúdico é composto por quatro elementos principais, que são: mecânica; estética; história/narrativa; e tecnologia. A seleção destes elementos se baseou nos trabalhos de Hunicke et al. 2004, Schell (2008), Aleven et al. (2010), Echeverría et al. (2011) e Villalta et al. (2011).

O processo de aplicação do modelo conceitual estabelece uma estrutura para a mediação do processo de ensino e aprendizagem de conteúdos. Para tanto, baseado em Schön (2000), é utilizado um plano para a aplicação do jogo, que apresenta os campos a serem preenchidos sobre o conhecimento do mediador na ação pedagógica, a reflexão para a ação pedagógica e a reflexão na ação pedagógica. As estratégias pedagógicas para o uso do jogo devem ser descritas no plano em acordo com os critérios da aprendizagem mediada de Feuerstein (1980), sendo estes adaptados em acordo com Klein, Nir-Gal e Darom (2000), Gomes (2001, 2002), Mentis (2002), Souza, Machado e Depresbiteris (2004), Campos e Macedo (2011) e ICELP (2012). Estes critérios são os mesmos apresentados para a criação, todavia voltados para mediação do aprendizado no conteúdo do jogo durante a prática pedagógica.

O processo de avaliação do modelo conceitual está estruturado por um plano de avaliação e por dois tipos de avaliações. Descrições e narrações, por meio da observação participante, para avaliar as estratégias pedagógicas (critérios de mediação de Feuerstein) utilizadas na prática pedagógica e a aplicação de três questionários para dar suporte para a coleta de dados para avaliação das variáveis reação e aprendizagem. O primeiro questionário é aplicado antes do jogo (pré-teste) para capturar a percepção dos jogadores a respeito de seu conhecimento no conteúdo do jogo; já o segundo e o terceiro deverão ser aplicados após o uso do jogo (pós-testes 01 e 02). O segundo questionário visa conhecer a reação do jogador sobre a sua experiência e motivação com o jogo e se os objetivos educacionais apregoados para o mesmo foram atingidos; e o terceiro questionário é para verificar, em comparação com o primeiro, se o jogo possibilitou melhoria do aprendizado no conteúdo do jogo. Sugere-se que as avaliações dos dados coletados dos questionários (pré e pós-testes 01 e 02) sejam analisadas por meio da estatística descritiva quantitativa para médias, utilizadas nas análises deste trabalho.

O quadro 1 apresenta o resumo da estrutura de avaliação do modelo conceitual.

\begin{tabular}{|c|c|c|}
\hline Variáveis & Construtos & Dimensões \\
\hline \multirow{13}{*}{ Reação } & \multirow[t]{4}{*}{ Motivação } & Atenção \\
\hline & & Relevância \\
\hline & & Confiança \\
\hline & & Satisfação \\
\hline & \multirow[t]{6}{*}{ Experiência do usuário } & Imersão \\
\hline & & Desafio \\
\hline & & Competência \\
\hline & & Divertimento \\
\hline & & Controle \\
\hline & & Interação social \\
\hline & \multirow[t]{3}{*}{ Objetivos educacionais do jogo } & Lembrar \\
\hline & & Entender \\
\hline & & Aplicar \\
\hline
\end{tabular}

V. $14 \mathrm{~N}^{\mathrm{o}} 2$, dezembro, 2016 


\begin{tabular}{|c|c|c|}
\hline & & Analisar \\
\hline & & Avaliar \\
\hline & & Criar \\
\hline \multirow[t]{5}{*}{ Aprendizagem } & $\begin{array}{l}\text { Conhecimentos no conteúdo (pré } \\
\text { e pós-teste) }\end{array}$ & $\begin{array}{l}\text { Depende do conteúdo e dos temas } \\
\text { apresentados pelo jogo }\end{array}$ \\
\hline & \multirow{4}{*}{$\begin{array}{l}\text { Estratégia pedagógica (critérios } \\
\text { para a aprendizagem mediada de } \\
\text { Feuerstein) }\end{array}$} & Intencionalidade e reciprocidade \\
\hline & & Significado \\
\hline & & Transcendência \\
\hline & & $\begin{array}{l}\text { Outros critérios da aprendizagem mediada } \\
\text { de Feuerstein utilizados e observados na } \\
\text { prática pedagógica (depende do uso dos } \\
\text { outros critérios) }\end{array}$ \\
\hline
\end{tabular}

Quadro 1. Estrutura do modelo conceitual para o processo de avaliação.

Fonte: Autores.

A estrutura está dividida em duas variáveis, cinco construtos e respectivas dimensões. Salienta-se que as dimensões do construto aprendizagem podem variar em função do conteúdo, temas e dos critérios da aprendizagem mediada utilizados na prática pedagógica.

\section{APLICAÇÃo E AVALIAÇão DO MODELO CONCEITUAL PARA JOGOS EDUCATIVOS DIGITAIS}

A aplicação e avaliação do modelo conceitual para a verificação da sua consistência seguiram os passos descritos para os processos de aplicação e avaliação do modelo, sendo estes considerados como os protocolos da coleta de dados da pesquisa. A estrutura proposta para o processo de criação do modelo conceitual foi concebida depois do desenvolvimento do Simulador Ambiental (SA) (jogo educativo digital), sendo este o ponto de entrada desta pesquisa, conforme apresenta o DSRM. Todavia, para fins de exemplificação da aplicabilidade desse processo, foi realizada uma análise crítica descritiva do "SA", comparando-se as recomendações do processo de criação com as características do "SA". A aplicação e a avaliação da consistência do modelo conceitual foram estruturadas na seguinte sequência:

- descrição do plano da aplicação e avaliação;

- preparação dos materiais (questionários, slides etc.);

- explicação do plano da aplicação ao professor da disciplina;

- apresentação do jogo e explicação dos questionários;

- aplicação do questionário (pré-teste);

- execução do jogo;

- aplicação dos questionários (pós-testes 01 e 02); e

- fechamento da prática pedagógica;

- análise dos dados.

Para a coleta e análise de dados, foi utilizada a observação participante para a aplicação e avaliação da prática pedagógica (plano da aplicação e avaliação) e de três questionários (pré-teste e pós-testes 01 e 02) sobre a percepção do usuário em relação ao jogo e a sua aprendizagem no conteúdo do jogo. Os itens dos questionários foram estabelecidos de acordo com o conteúdo do "SA", que trata dos hábitos de consumo e produção no ambiente residencial. Esses hábitos estão relacionados à sustentabilidade no ambiente residencial, sendo caracterizados, principalmente, pelo consumo de 
energia, consumo de água e a produção de resíduos líquidos e sólidos oriundos de uma edificação (ARBEX et al., 2012).

A aplicação do modelo conceitual para verificar sua consistência foi realizada na Faculdade Senac Florianópolis, nos laboratórios de informática da instituição, com quatro turmas do projeto Jovens Aprendizes, e três turmas do programa Pronatec. No total, participaram 125 alunos distribuídos por sete turmas, em horários distintos. A faixa média de idade das sete turmas ficou em torno de 14 a 16 anos. As turmas dos Jovens aprendizes apresentam médias de idade mais baixas em relação às turmas do Pronatec, haja visto que das turmas do Pronatec (327, 456 e 757) fazem parte alguns alunos com idade superior a 30 anos

Os planos das aplicações demonstraram ter consistência na sistemática desenvolvida para os mesmos, pois ajudaram a desenvolver o planejamento, operação e análise das práticas pedagógicas com o uso do jogo em sala de aula. A utilização dos planos da aplicação nas práticas pedagógicas ajudou a definir as reflexões para a ação (planejamento da prática pedagógica), postulado de Schön (2000) sobre o modelo crítico reflexivo da prática docente, assim como para os registros e anotações, por parte dos mediadores (professores e pesquisador), para a reflexão na ação, ou seja, as observações e ações executadas durante as práticas pedagógicas. Portanto, os planos das aplicações demonstraram ter consistência na sistemática desenvolvida para os alunos, pois apoiam o planejamento, operação e análise durante as práticas pedagógicas com o uso do jogo em sala de aula.

Os planos das avaliações, também com base em Schön (2000), possibilitaram a reflexão sobre a ação pedagógica, principalmente no que diz respeito ao cronograma das atividades para a coleta de dados e na estruturação das análises dos resultados finais das práticas pedagógicas, assim como dos registros e anotações da reflexão sobre a ação pedagógica, no que se refere às análises dos resultados alcançados com a estratégia pedagógica definida, da percepção dos alunos sobre o jogo e sua aprendizagem, culminando com as sugestões de melhoria, tanto para o jogo, quanto para a estratégia da prática pedagógica.

A estratégia pedagógica planejada, apoiada nos critérios da aprendizagem mediada de Feuerstein (1980) (ver proposta), para as práticas pedagógicas foi positiva, todos os critérios fundamentais para a aprendizagem mediada foram trabalhados. Podese destacar que os três critérios fundamentais, formalmente descritos nos planos de aplicação, foram atingidos.

- Intencionalidade e reciprocidade - As práticas pedagógicas com uso do "SA" sensibilizaram os participantes para a importância de práticas sustentáveis de consumo e produção no ambiente residencial. Alguns problemas verificados, como a gestão do tempo, velocidade da internet e o entendimento dos participantes quanto aos passos a serem seguidos para jogar, não interferiram na reciprocidade dos alunos.

- Significado - os participantes demonstraram terem entendido a razão da atividade de aprendizagem e a importância das tarefas realizadas. O planejamento das atividades valorizou as atitudes e habilidades dos alunos, tais como: cooperação, argumentação, disciplina, respeito aos colegas, etc.

- Transcendência - Os ambientes do jogo (SA) e as possibilidades de ação ajudaram os participantes a fazerem analogias e debates sobre cotidiano vivido em suas 
residências com as situações apresentadas pelo jogo, desencadeando intenções para atitudes mais sustentáveis nas suas residências.

Ressalta-se que os demais critérios da aprendizagem mediada de Feuerstein (1980) também foram trabalhados pelos mediadores (professores); mesmo que de forma não planejada, eles foram utilizados em algum momento durante as práticas pedagógicas.

Constatou-se, em referência aos resultados gerais obtidos por meio da análise das respostas, com o uso da estatística descritiva, nas sete práticas pedagógicas, que houve uma melhoria significativa da percepção dos alunos quanto ao seu aprendizado no conteúdo do jogo (SA). Em relação à adequação do jogo ao público infanto-juvenil, a aplicação do "SA" demonstrou estar adequado à ZDP dos alunos, postulado de Vygotsky (2001). Todavia, a aplicação do jogo também foi efetiva nas turmas 327 e 757, que apresentaram uma média maior de idade. Esta constatação de melhoria na aprendizagem pode ser afirmada pelos resultados gerais obtidos no pré-teste e pós-testes 01 e 02 , principalmente em relação aos somatórios das médias obtidas dos quadrantes negativos e positivos, e pelos valores obtidos para os níveis de significância $(\alpha)$, sendo $\mathrm{p}=0,2 \%$ para as médias negativas e $\mathrm{p}=0,62 \%$ para as médias positivas.

Portanto, de acordo com os somatórios e médias gerais apresentadas e confirmadas pelos cálculos gerais dos níveis de significância $(\alpha)$, pode-se extrapolar estatisticamente, para toda a população, a afirmação de que o jogo (SA) oportuniza o aprendizado para a sustentabilidade no ambiente residencial, conteúdo do jogo. Também se pode afirmar, considerando as respostas por questão, que o jogo leva à aprendizagem nas dimensões do conteúdo do "SA". Nesse sentido, estatisticamente, considerando os cálculos dos níveis de significância $(\alpha)$ para cada questão arguida no pré-teste e pós-teste 02 , pode-se extrapolar que o jogo ajuda na reflexão sobre os hábitos de consumo e produção no ambiente residencial, especialmente no que diz respeito a suas dimensões: consumo de energia elétrica, consumo de água, produção de resíduos sólidos e líquidos.

\section{CONSIDERAÇÕES FINAIS}

Este trabalho buscou em evidências teóricas e empíricas, aprimorar o conhecimento das pessoas sobre os jogos educativos digitais, a criação e a didática de aplicação destes em sala de aula, assim como a avaliação da qualidade e aprendizagem. A estrutura desenvolvida para os processos do modelo conceitual foi concebida por meio dos procedimentos metodológicos do DSRM, de acordo com Peffers et al. (2007), sendo o "SA" o ponto de entrada ou início da pesquisa, conforme apresenta a metodologia. A partir da experiência aprendida com o desenvolvimento do jogo, concomitantemente com os avanços das pesquisas bibliométricas e aleatórias, estruturou-se o modelo conceitual deste trabalho.

Nesse sentido, com base nos resultados da verificação da consistência, contatouse que o processo de criação do modelo conceitual possibilita que os desenvolvedores de jogos tenham melhor clareza dos elementos pedagógicos e lúdicos a serem concebidos no design instrucional do jogo. Referente à estrutura pedagógica, o modelo conceitual demonstrou que a utilização da taxionomia de Bloom revisada apoia a definição dos objetivos educacionais do jogo. Assim como, o guia com os critérios de 
mediação de Feuerstein possibilita a concepção de estratégias pedagógicas que facilitem a mediação do processo de ensino e aprendizagem em sala de aula com o uso de um jogo educativo digital.

O processo de aplicação do modelo conceitual demonstrou, com referência aos registros da observação participante e resultados apresentados, ser útil na definição das estratégias pedagógicas para a mediação do processo de ensino e aprendizagem de conteúdos ligados a um jogo educativo digital. Os planos das aplicações, com base Schön (2000), demonstraram ter consistência na sistemática desenvolvida para os mesmos, pois possibilitaram uma estrutura para o planejamento, operação e análise durante as práticas pedagógicas, com o uso do jogo em sala de aula.

Também se constatou a utilidade do processo de avaliação do modelo conceitual, pois trouxe uma estrutura para a avaliação dos jogos e de reflexões sobre a ação pedagógica, o que ajudou na verificação da consistência da efetividade do modelo conceitual. Por meio dos planos de avaliação e dos questionários desenvolvidos, foi possível avaliar a reação (motivação, experiência do usuário e objetivos educacionais do jogo) e a aprendizagem dos jogadores (conhecimentos no conteúdo e a estratégia pedagógica utilizada).

\section{REFERÊNCIAS}

ALEVEN, V. et al. Toward a framework for the analysis and design of educational games. In: IEEE - INTERNATIONAL CONFERENCE ON DIGITAL GAME AND INTELLIGENT TOY ENHANCED LEARNING, p. 69-76. Proceedings, 2010.

ANDERSON, L. W. et al. A taxonomy for learning, teaching, and assessing: a revision of Bloom's taxonomy of educational objectives. New York: Longman, 2001.

ARBEX, D.; JAPPUR, R.; SELIG, P.; \& VARVAKIS, G. Ergonomic aspects simulation digital online: an educational game proposal to promote environmental education. Work: A Journal of Prevention, Assessment and Rehabilitation, v. 41, p. 6011-6015, 2012. Disponível em: <http://iospress.metapress.com/content/?k=jappur $\geq$. Acesso em: 01 mar. 2012. doi:10.3233/WOR-2012-1052-6011.

BAEK, Y. What hinders teachers in using computer and video games in the classroom? Exploring factors inhibiting the uptake of computer and video games. $\begin{array}{lllllllll}\text { Cyberpsychology } \& \text { Behavior, } & \text { v. 11, } & \mathrm{n}^{\circ} & 6, & \text { p. } & 665-671, & \end{array}$ doi:10.1089/cpb.2008.0127.

BALASUBRAMANIAN, N.; WILSON, B. G. Games and simulations. In: SOCIETY FOR INFORMATION TECHNOLOGY AND TEACHER EDUCATION INTERNATIONAL CONFERENCE, 2006. Proceedings, v. 1, 2006. Disponível em: $<$ http://site.aace.org/pubs/foresite/GamesAndSimulations1.pdf $>$. Acesso em: 08 jan. 2012.

BLOOM, B. S.; ENGELHART M. D.; FURST, E. J.; HILL, W. H.; \& KRATHWOHL, D. R. Taxionomia de objetivos educacionais: domínio cognitivo. Porto Alegre: Editora Globo, $6^{\circ}$ ed., 1977. 
CAMPOS, M. C. R. M.; MACEDO, L. Desenvolvimento da função mediadora do professor em oficinas de jogos. Revista Semestral da Associação Brasileira de Psicologia Escolar e Educacional. São Paulo: v. 15, n 2, p. 211-220. Jul/Dez., 2011. doi:10.1590/S1413- 85572011000200003.

ECHEVERRÍA, A.; GARCÍA-CAMPO, C.; NUSSBAUM, M.; GIL, F.; VILLALTA, M.; AMÉSTICA, M.; \& ECHEVERRÍA, S.. A framework for the design and integration of collaborative classroom games. Computers \& Education, v. 57, p. 1127-1136, 2011. Disponível em: <www.elsevier.com/locate/compedu>. Acesso em: 10 mar. 2012. doi:10.1016/j. compedu.2010.12.010.

FEUERSTEIN, R. et al. Instrumental enrichment: an intervention program for cognitive modifiability. Glenview (Illinois): Scott, Foresman and Company, 1980.

GIL, Antonio Carlos. Como elaborar projetos de pesquisa. 5. ed. São Paulo, SP: Atlas, 2010.

GOMES, C. M. A. Em busca de um modelo psico-educativo para a avaliação de softwares educacionais. Dissertação (Mestrado em Engenharia de Produção) Programa de Pós-Graduação em Engenharia de Produção, Universidade Federal de Santa Catarina, Florianópolis, 2001. Disponível em: <http://aspro02.npd.ufsc.br/pergamum/biblioteca/index.php?resolution2= 024_1\&tipo_pesquisa=\#posicao_dados_acervo>. Acesso em: 15 fev. 2012. $\overline{\text { Ed. } 2002 .}$

Feuerstein e a construção mediada do conhecimento. Porto Alegre: Artmed

HSIAO, Hui-Chun. A brief review of digital games and learning. Digitel 2007, pp. 124127. In: THE FIRST IEEE INTERNATIONAL WORKSHOP ON DIGITAL GAME AND INTELLIGENT TOY ENHANCED LEARNING (DIGITEL'07). Proceedings Los Alamitos, CA, USA: IEEE Computer Society, 2007. Disponível em: <http://doi.ieeecomputersociety.org/10.1109/DIGITEL.2007.3>. Acesso em $15 \mathrm{dez}$. 2012. doi:10.1109/DIGITEL.2007.3.

HUNICKE, R.; LEBLANC, M.; \& ZUBEK, R. MDA: A formal approach to game design and ame research. In: FU, D.; HENKE, S.; ORKIN, J. (Eds.). Challenges in game artificial intelligence. Papers from the 2004 AAAI WORKSHOP TECHNICAL REPORT WS-04-04. Menlo Park, CA: The AAAI Press, p. 1-5, 2004.

ICELP - The International Center for the Enhancement of Learning Potential. Research: basic theory. Disponível em: <www.icelp.org/asp/Basic_Theory.shtm.>. Acesso em: 26 jun. 2012.

KIRRIEMUIR, J; MCFARLANE, A. Literature review in games and learning. Bristol: Futurelab, 2004. Disponível em: <www.mendeley.com/research/literaturereview-in-games-and-learning/>. Acesso em: 05 jan. 2012.

KLEIN, S. P.; NIR-GAL, O.; \& DAROM, E. The use of computers in kindergarten, with or without adult mediation; effects on children's cognitive performance and 
behavior. Computers in Human Behavior, 16(6), 591-608, 2000. doi:10.1016/S07475632(00)00027-3.

KRATHWOHL. D. R. A revision of bloom's taxonomy: an overview. Theory into Practice, V. 41, n. 4, Autumn, p. 212-264, 2002. Disponível em: <www.celt.iastate.edu/teaching/RevisedBlooms1.html>. Acesso em: 26 de jun. 2012.

MAYER, Richard E. A taxonomy for computer-based assessment of problem solving. Computers in Human Behavior, v. 18, p. 623-632, 2002.

MENTIS, M. Aprendizagem mediada dentro e fora de sala de aula. Programa de pesquisa cognitiva. Divisão de educação especializada da universidade de Witwatersand, África do Sul. $3^{\circ}$ Ed. São Paulo: Instituto Pieron de Psicologia Aplicada, 2002.

MORENO-GER, P., BURGOS, D., MARTÍNEZ-ORTIZ, I., SIERRA, J. L., \& FERNÁNDEZ-MANJÓNA, B. Educational game design for online education.

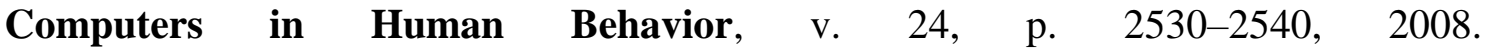
doi:10.1016/j.chb.2008.03.012.

PEFFERS, K.; TUUNANEN, T.; ROTHENBERGER, M. A.; \& CHATTERJEE, S. A design science research methodology for information systems research. Journal of Management Information Systems, v. 24, n. 3, p. 45-77. Doi: 10.2753/MIS0742$1222240302,2007$.

SAVI, Rafael. Avaliação de jogos voltados para a disseminação do conhecimento. Tese (Doutorado em Engenharia e Gestão do Conhecimento) - Programa de PósGraduação em Engenharia e Gestão do Conhecimento, Universidade Federal de Santa Catarina, Florianópolis, 2011. Disponível em: <http://btd.egc.ufsc.br/?p=1038>. Acesso em: 15 jan. 2012.

SCHELL, J. The art of game design: a book of lenses. Morgan Kaufmann. 2008.

SCHON, D. A. Educando o profissional reflexivo: um novo design para o ensino e a aprendizagem. Artmede, Porto Alegre, 2000.

SOUZA, A. M. M.; MACHADO, O. T. M.; \& DEPRESBITERIS, L. A mediação como princípio deducional: Bases teóricas das abordagens de Reuven Feuerstein. São Paulo: Senac, 2004.

VERGARA, S. C. Projetos e relatórios de pesquisa em administração. 13. ed. São Paulo, SP: Atlas, 2009.

VILLALTA. M.; GAJARDO, I.; NUSSBAUM. M.; ANDREU, J. J.; ECHEVERRÍA, A.; \& PLASS, J. L. Design guidelines for classroom multiplayer presential games (CMPG). Computers \& Education, v. 57, p. 2039-2053, 2011. Disponível em: <www.elsevier.com/locate/compedu>. Acesso em: 10 de mar. de 2012. doi:10.1016/j.compedu.2011.05.003.

VYGOTSKY, L. S. A construção do pensamento e da linguagem. São Paulo, Martins Fontes, 2001. 